\title{
Microestrutura, fases cristalinas e propriedades elétricas de porcelanas aluminosas contendo diferentes concentrações de $\mathrm{Fe}_{2} \mathrm{O}_{3}$ sinterizadas em atmosfera redutora e oxidante
}

\author{
(Microstructure, crystalline phases and electrical properties of \\ aluminous porcelain containing different $\mathrm{Fe}_{2} \mathrm{O}_{3}$ concentrations sintered \\ in reducing and oxidizing atmosphere)
}

\author{
D. H. Pival, ${ }^{1,2}$, R. H. Piva ${ }^{1}$, J. Venturini ${ }^{2}$, M. R. Morelli, C. P. Bergmann ${ }^{2}$ \\ ${ }^{1}$ Laboratorio de Formulação e Sintese Ceramica, Universidade Federal de S. Carlos, S. Carlos, SP, Brasil \\ ${ }^{2}$ Laboratório de Materiais Cerâmicos, Universidade Federal do Rio Grande do Sul, Porto Alegre, RS, Brasil
}

\begin{abstract}
Resumo
Porcelanas aluminosas contendo diferentes concentrações de $\mathrm{Fe}_{2} \mathrm{O}_{3}$ foram sinterizadas em atmosfera oxidante e redutora. A microestrutura e fases formadas foram investigadas com o auxílio de difração de raios X e microscopia eletrônica de varredura. Espectroscopia de impedância foi utilizada para estudar as propriedades elétricas. Os resultados indicaram que todas as amostras apresentaram mulita e coríndon como fases majoritárias e pequenas frações de quartzo. A adição de $\mathrm{Fe}_{2} \mathrm{O}_{3}$ provocou o surgimento de hematita nas amostras sinterizadas em atmosfera oxidante e, ferro metálico nas amostras contendo $>3 \%$ p sinterizadas em atmosfera redutora. Os espectros de impedância indicam que as características da fase vítrea e interface entre fase vítrea /fases cristalinas regem o comportamento elétrico global das amostras. Não obstante, diferentes atmosferas de queima provocam efeitos opostos na resistividade elétrica das porcelanas contendo $\mathrm{Fe}_{2} \mathrm{O}_{3}$. A presença de hematita foi considerada a responsável pela diminuição da resistividade elétrica nas amostras sinterizadas em atmosfera oxidante, enquanto que o aumento na resistividade elétrica das amostras sinterizadas em atmosfera redutora foi atribuído à elevada quantidade de fase vítrea e porosidade. Estes resultados indicam que atmosfera redutora durante a queima pode favorecer o uso de matérias-primas com maiores concentrações de $\mathrm{Fe}_{2} \mathrm{O}_{3}$, desde que todo óxido de ferro tenha se dissolvido na fase liquida durante a queima.

Palavras-chave: porcelana, espectroscopia de impedância, $\mathrm{Fe}_{2} \mathrm{O}_{3}$, resistividade elétrica.
\end{abstract}

Abstract

Aluminous porcelain containing different $\mathrm{Fe}_{2} \mathrm{O}_{3}$ concentrations were sintered in oxidizing and reducing atmosphere. The microstructure and crystalline phase formation were investigated by scanning electron microscopy and X-ray diffraction. Electrical properties were investigated by Impedance spectroscopy. The results indicated that all samples showed mullite and corundum as major phases and small fractions of quartz. The addition of $\mathrm{Fe}_{2} \mathrm{O}_{3}$ caused the appearance of hematite in samples sintered in oxidizing atmosphere and metallic iron for the samples containing $>3 \% w t$. and sintered in reducing atmosphere. The impedance spectrum indicates that the glass phase and crystal/glassy phase interface govern the overall electrical behavior of the samples. However, different firing atmospheres cause opposite effect on the electrical resistivity of the porcelain containing $\mathrm{Fe}_{2} \mathrm{O}_{3}$. The presence of hematite was considered to be responsible for the decrease in resistivity for samples sintered in oxidizing atmosphere, whereas the increase in the resistivity of the sample sintered in a reducing atmosphere was attributed to the high amount of glassy phase and porosity. These results indicate that reducing atmosphere during firing may favor the use of raw materials with higher concentrations of $\mathrm{Fe}_{2} \mathrm{O}_{3}$, since all iron oxide have been dissolved into the melting phase during the sintering of porcelain bodies. Keywords: porcelain, impedance spectroscopy, $\mathrm{Fe}_{2} \mathrm{O}_{3}$, electrical resistivity.

\section{INTRODUÇÃO}

Porcelanas triaxiais têm sido por década utilizadas como isoladores elétricos. Essa hegemonia se da devido as suas propriedades dielétricas combinadas com suas excelentes propriedades mecânicas e, principalmente, a capacidade de manutenção dessas propriedades em condições severas, como: umidade e alta temperatura [1]. De uma maneira geral, as porcelanas elétricas são essencialmente compostas de caulim e/ou argila, feldspato e quartzo [2]. Frequentemente, em porcelanas de alta tensão, o quartzo é parcialmente ou totalmente substituído por coríndon para aumentar a resistência mecânica do produto final [3]. Quando tratada termicamente de forma adequada, essas matérias-primas formam uma matriz vítrea com cristais de quartzo/coríndon, mulita e porosidade remanescente [4]. A ação sinérgica destas fases é responsável pelas propriedades acima mencionadas, ao passo que uma adequada seleção das matérias-primas é de suma importância para as características finais do produto.

Um dos pré-requisitos na seleção de matérias-primas 
para a produção de isoladores elétricos de porcelana é a baixa concentração de óxidos de metais de transição conhecidos como impurezas, tais como $\mathrm{TiO}_{2}, \mathrm{Fe}_{2} \mathrm{O}_{3}, \mathrm{Cr}_{2} \mathrm{O}_{3}$ [1]. A presença desses óxidos altera o comportamento elétrico do produto final, provocando principalmente uma redução das propriedades de isolamento devido à condução eletrônica por "small polaron hopping" entre íons com diferentes estados de valência [5]. Nas últimas décadas, vários trabalhos sobre porcelanas têm sido realizados para verificar a relação entre a composição química das matériasprimas e as propriedades elétricas resultantes [2, 6-10]. No entanto, pouco se sabe sobre a dependência das propriedades elétricas de porcelanas contendo essas impurezas quando submetidos a diferentes atmosferas de queima.

Entre os óxidos acima mencionados, o $\mathrm{Fe}_{2} \mathrm{O}_{3}$ se destaca como a impureza mais comum e indesejável presente nas matérias-primas. Porém, estudos que abordam a influência de sua concentração sobre as propriedades elétricas de porcelanas são escassos na literatura. Em um desses poucos trabalhos, Piva et al. [11] observou que o efeito deletério na constante dielétrica e tangente de perdas causado pela presença do $\mathrm{Fe}_{2} \mathrm{O}_{3}$ está vinculado a não solubilização deste óxido nas fases da porcelana. De acordo com seus resultados, a utilização de matérias-primas com maiores concentrações de $\mathrm{Fe}_{2} \mathrm{O}_{3}$ do que a atualmente utilizada não traria grandes alterações nas propriedades dielétricas, porém possibilitaria ganhos econômicos significativos em relação à aquisição de insumos e na etapa de sinterização, uma vez que a presença deste óxido reduz o preço das matérias-primas e diminui a temperatura de queima [12].

Portanto, devido à ausência de informação sobre o assunto na literatura e a relevância do tema para a compreensão do efeito de uma das impurezas mais comuns presentes nas matérias-primas para a indústria de isoladores de porcelanas, este trabalho apresenta resultados quanto ao efeito da concentração de $\mathrm{Fe}_{2} \mathrm{O}_{3}$ sobre as propriedades elétricas de porcelanas aluminosas sinterizadas em atmosferas oxidante e redutora.

\section{MATERIAIS E MÉTODOS}

Inicialmente, uma formulação denominada padrão (STD) com baixo teor de $\mathrm{Fe}_{2} \mathrm{O}_{3}$ e pertencente ao grupo das porcelanas aluminosas [13] foi obtida através da mistura das matérias-primas feldspato:caulim:alumina (fornecido pela

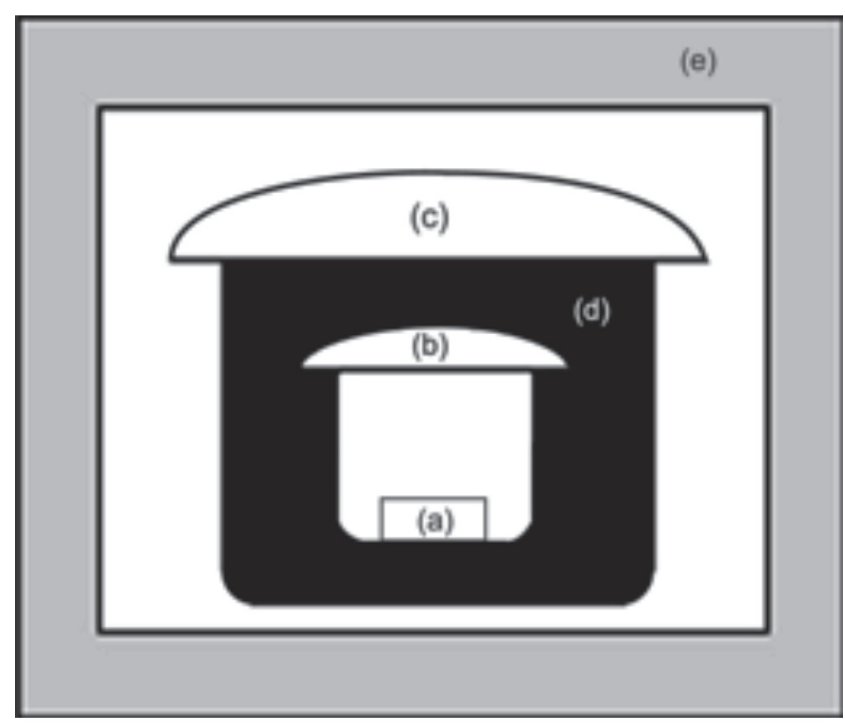

Figura 1: Representação da "cama de grafite" desenvolvida para criar a condição atmosférica redutora durante a etapa de queima. (a) amostra; (b) e (c) cadinhos de alumina; (d) pó de grafite e (e) forno elétrico.

[Figure 1: "Graphite bed" representation designed to create the reducing atmospheric condition during the firing step. (a) sample; (b) and (c) alumina crucibles; (d) graphite powder and (e) electric furnace.]

Universidade de Aveiro, Portugal) na proporção 30:30:40. Para verificar o efeito da concentração de $\mathrm{Fe}_{2} \mathrm{O}_{3}$ sobre as propriedades elétricas das porcelanas, quatro formulações foram elaboradas a partir da formulação STD, com adição progressiva de 0, 3, 5 e 8\%peso de $\mathrm{Fe}_{2} \mathrm{O}_{3}(99,9 \%$, SigmaAldrich) denominados P0, P3, P5 e P8, respectivamente. A Tabela I apresenta as análises químicas das formulações utilizadas, obtidas por fluorescência de raios X (Philips PW 2400).

Uma vez que todas as matérias-primas eram industrialmente beneficiadas, as formulações foram misturadas por via úmida em um moinho planetário por 20 min. Após a homogeneização as suspensões foram secas a $110^{\circ} \mathrm{C}$ até completa remoção da água, desaglomeradas para obtenção dos pós e posteriormente umidificado com 7\%peso de água. Corpos de prova cilíndricos $(10 \mathrm{~mm}$ de diâmetro x $3 \mathrm{~mm}$ de espessura) foram obtidos através de prensagem uniaxial com $125 \mathrm{MPa}$. Por fim, as amostras foram secas a $110{ }^{\circ} \mathrm{C}$ por $24 \mathrm{~h}$.

Tabela I - Composição química das formulações utilizadas.

[Table I-Chemical composition of the used formulations.]

\begin{tabular}{ccccccccccc}
\hline \multirow{2}{*}{ Formulações } & $\mathrm{Al}_{2} \mathrm{O}_{3}$ & $\mathrm{SiO}_{2}$ & $\mathrm{Fe}_{2} \mathrm{O}_{3}$ & $\mathrm{CaO}$ & $\mathrm{Na}_{2} \mathrm{O}$ & $\mathrm{K}_{2} \mathrm{O}$ & $\mathrm{MnO}$ & $\mathrm{MgO}$ & $\mathrm{TiO}_{2}$ & $\mathrm{P}_{2} \mathrm{O}_{5}$ \\
\hline $\mathrm{P} 0$ & 57,38 & 38,84 & 0,29 & 0,26 & 1,74 & 1,20 & 0,01 & 0,03 & 0,08 & 0,17 \\
$\mathrm{P} 3$ & 55,60 & 37,63 & 3,39 & 0,26 & 1,68 & 1,17 & 0,01 & 0,03 & 0,08 & 0,17 \\
P5 & 54,41 & 36,83 & 5,44 & 0,25 & 1,65 & 1,14 & 0,01 & 0,03 & 0,08 & 0,16 \\
P8 & 52,64 & 35,63 & 8,52 & 0,24 & 1,59 & 1,11 & 0,01 & 0,03 & 0,07 & 0,16 \\
\hline
\end{tabular}


Tabela II - Parâmetros utilizados durante a queima e respectivos códigos das amostras.

[Table II - Fired conditions and codes of the samples.]

\begin{tabular}{lcccc}
\hline Formulações & P0 & P3 & P5 & P8 \\
\hline Código para as amostras queimadas em atmosfera oxidante $\left(\mathrm{O}_{2}\right)$ & $\mathrm{P} 0 \mathrm{O}$ & $\mathrm{P} 3 \mathrm{O}$ & $\mathrm{P} 5 \mathrm{O}$ & $\mathrm{P} 8 \mathrm{O}$ \\
Código para as amostras queimadas em atmosfera redutora $\left(\mathrm{CO} / \mathrm{CO}_{2}\right)$ & $\mathrm{P} 0 \mathrm{R}$ & $\mathrm{P} 3 \mathrm{R}$ & $\mathrm{P} 5 \mathrm{R}$ & $\mathrm{P} 8 \mathrm{R}$ \\
Temperatura máxima de queima $\left({ }^{\circ} \mathrm{C}\right)$ & 1300 & 1250 & 1200 & 1200 \\
Taxa de aquecimento/ resfriamento $\left({ }^{\circ} \mathrm{C} \mathrm{min}^{-1}\right)$ & 10 & 10 & 10 & 10 \\
Patamar (min.) & 120 & 120 & 120 & 120 \\
\hline
\end{tabular}

Para verificar o efeito da atmosfera de queima, os corpos de prova foram divididos em dois grupos. O primeiro grupo foi queimado em um forno elétrico hermeticamente isolado com atmosfera rica em $\mathrm{O}_{2}$ para criar a condição oxidante. $\mathrm{O}$ segundo grupo foi queimado em um forno elétrico sob atmosfera de $\mathrm{CO} / \mathrm{CO}_{2}$ para criar a condição redutora. Para este caso, foi utilizado o aparato conhecido como "cama de grafite" [14]. Inicialmente as amostras foram acomodadas no interior de um pequeno cadinho de alumina fechado, este então foi introduzido no interior de um cadinho maior e completamente recoberto com pó de grafite, o esquema é representado na Fig. 1. O conjunto de cadinhos foi então aquecido até a temperatura desejada.

As temperaturas máximas de queima para as formulações estudadas foram definidas com base em um trabalho anterior [11]. Buscou-se manter as mesmas temperaturas para as mesmas formulações para que a atmosfera fosse à única variável durante a queima. A Tabela II apresenta as condições utilizadas para a sinterização dos corpos-deprova e os códigos que serão utilizados para identificação das amostras.

A absorção de água (AA) das amostras foi determinada de acordo com a seguinte Equação:

$$
\mathrm{AA}=\left(\frac{\mathrm{Pu}-\mathrm{Ps}}{\mathrm{Ps}}\right) \times 100
$$

na qual Ps e Pu são o peso seco e o peso úmido da amostra, respectivamente.

As fases cristalinas formadas durante a sinterização foram identificadas por difração de raios X. O ensaio foi realizada em um difratômetro Philips X'Pert MPD utilizando uma fonte de radiação $\mathrm{Cu}-\mathrm{k} \alpha(1,5406 \AA)$, operando em $40 \mathrm{kV}$ e $40 \mathrm{~mA}$. Os difratogramas foram obtidos na faixa de 10 a $90^{\circ}$ (20) com um passo de $0,05^{\circ}$. As fases foram identificadas utilizando o software X-Pert plus. Amostras sinterizadas foram polidas e atacadas com uma solução de ácido fluorídrico durante 1 min para observação da microestrutura por microscopia eletrônica de varredura (Jeol JEM 2010) sob uma tensão de aceleração de $20 \mathrm{kV}$.

As propriedades elétricas foram obtidas por espectroscopia de impedância (Metrohm Autolab PGSTAT30) com um analisador de resposta de frequência FRA32M. A faixa de frequência de varredura foi de 0,1 $\mathrm{Hz}$ a $1 \mathrm{MHz}$ com uma voltagem de 0,5 V. Os diagramas de impedância foram analisados com o software Autolab
Nova. Pasta de prata foi aplicada em ambas as superfícies da amostra como contatos. As amostras foram posteriormente submetidas a um tratamento térmico a $400{ }^{\circ} \mathrm{C}$ por 15 min para melhorar a aderência da prata. As medidas de espectroscopia de impedância foram realizadas em ar atmosférico de 25 a $700{ }^{\circ} \mathrm{C}$ com uma medida a cada $50^{\circ} \mathrm{C}$. A taxa de aquecimento foi $10{ }^{\circ} \mathrm{C} \mathrm{min}{ }^{-1}$ e estabeleceu-se um patamar de 10 min antes de cada medida com o objetivo de reduzir variações de temperatura nas amostras.

A resistividade elétrica $(\rho)$ das amostras foi calculada por meio da equação:

$$
\rho=\frac{A \cdot R_{\text {total }}}{L}
$$

na qual A e L são a área e a espessura da amostra, respectivamente, e $\mathrm{R}_{\text {total }}$ é a resistência elétrica total obtida no espectro de impedância

A dependência da resistividade com a temperatura foi observada por meio do gráfico de Arrhenius $\left(\log _{10} \rho \times 1 / T\right)$. A energia de ativação foi determinada por meio da equação de Arrhenius:

$$
\rho=\rho_{0} \exp \left(\frac{\mathrm{Ea}}{\mathrm{k} \cdot \mathrm{T}}\right)
$$

na qual $\rho_{\mathrm{O}}, \mathrm{E}_{\mathrm{a}}, k$ e $\mathrm{T}$ são, respectivamente, fator préexponencial, energia de ativação, constante de Boltzmann e temperatura absoluta.

\section{RESULTADOS E DISCUSSÃO}

Na Fig. 2 são apresentados os difratogramas de raios $\mathrm{X}$ das porcelanas contendo diferentes concentrações de $\mathrm{Fe}_{2} \mathrm{O}_{3} \mathrm{e}$ sinterizadas em atmosfera de $\mathrm{O}_{2}$ e CO/CO . Todas as amostras apresentam um "background" entre os ângulos 20-30 (20) característico de fases amorfas [15], indicando elevada presença de fase vítrea. Para as amostras sinterizadas em atmosfera de $\mathrm{O}_{2}$ (Fig. 2a), coríndon e mulita apresentaramse como fases majoritárias e pequena fração de quartzo foi observada. A adição de $\mathrm{Fe}_{2} \mathrm{O}_{3}$ resultou no surgimento de uma quarta fase, hematita. De acordo com um trabalho anterior [11], em elevada temperatura, baixas concentrações de $\mathrm{Fe}_{2} \mathrm{O}_{3}$ são normalmente dissolvidas na fase liquida da porcelana devido à formação de um eutético durante a queima. No entanto, os resultados de difração de raios X mostram 

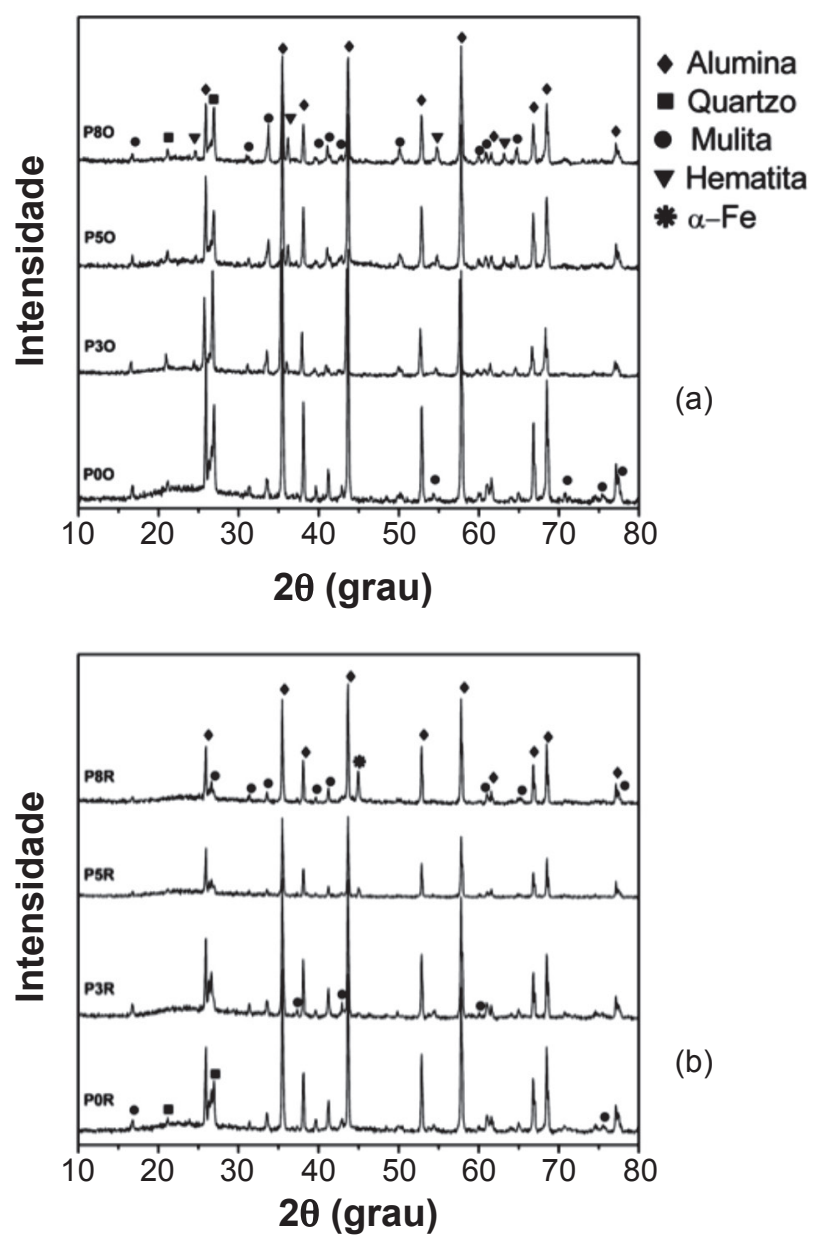

(b)

Figura 2: Difratogramas de raios $\mathrm{X}$ das formulações sinterizadas em atmosfera de (a) $\mathrm{O}_{2}$ e (b) $\mathrm{CO} / \mathrm{CO}_{2}$.

[Figure 2: XRD patterns of formulations sintered at atmosphere of (a) $\mathrm{O}_{2}$ and (b) $\mathrm{CO} / \mathrm{CO}_{2}$.]

que o limite de solubilidade do $\mathrm{Fe}_{2} \mathrm{O}_{3}$ na fase liquida foi alcançado, mesmo para a formulação contendo apenas 3 \%peso de $\mathrm{Fe}_{2} \mathrm{O}_{3}$. Os difratogramas das amostras sinterizadas em atmosfera de $\mathrm{CO} / \mathrm{CO}_{2}$ são apresentados na Fig. 2b. As amostras apresentaram as fases mulita e coríndon como fases majoritárias e quartzo como fase residual. Para as formulações contendo concentração maior que $3 \%$ peso de $\mathrm{Fe}_{2} \mathrm{O}_{3}$ (P5R e P8R) a fase $\alpha-\mathrm{Fe}$ (ferro metálico) foi observada. Vale ressaltar que houve uma redução na intensidade de difração dos picos da fase quartzo para as amostras com maior concentração de $\mathrm{Fe}_{2} \mathrm{O}_{3}$ sinterizado em atmosfera de $\mathrm{CO} / \mathrm{CO}_{2}$.

É de conhecimento que em condições redutoras a hematita reduz para wustita $(\mathrm{FeO})$ conforme reações descrito pelas equações D e E. Se a condição persistir, a reação descrita pela equação $\mathrm{F}$ pode ocorrer, onde todo $\mathrm{o} \mathrm{FeO}$ é reduzido à ferro metálico.

$$
\begin{aligned}
& 3 \mathrm{Fe}_{2} \mathrm{O}_{3}+\mathrm{CO} \rightarrow 2 \mathrm{Fe}_{3} \mathrm{O}_{4}+\mathrm{CO}_{2} \\
& \mathrm{Fe}_{3} \mathrm{O}_{4}+\mathrm{CO} \rightarrow 3 \mathrm{FeO}+\mathrm{CO}_{2} \\
& \mathrm{FeO}_{3}+\mathrm{CO} \rightarrow \mathrm{Fe}+\mathrm{CO}_{2}
\end{aligned}
$$

Assim, nas amostras contendo óxido de ferro e sinterizadas em atmosfera de $\mathrm{CO} / \mathrm{CO}_{2}$ todo $\mathrm{o} \mathrm{Fe}_{2} \mathrm{O}_{3}$ foi inicialmente reduzido para $\mathrm{FeO}$. Durante a etapa de queima o FeO reage com a sílica formando faialita $\left(2 \mathrm{FeO} \cdot \mathrm{SiO}_{2}\right)$ [16]. Entretanto, a aproximadamente $1170{ }^{\circ} \mathrm{C}$ a faialita irá formar eutéticos com o quartzo e $\mathrm{FeO}$ remanescentes, produzindo grandes quantidade de fase líquida. Além disso, uma vez dissolvido na fase líquida, o FeO fornece íons $\mathrm{Fe}^{2+}$ que atuam como modificadores de rede provocando uma diminuição da viscosidade da fase líquida [17] e, consequentemente, atacando agressivamente pequenos grãos de quartzo, o que explica a diminuição nos picos de quartzo nas amostras contendo inicialmente $\mathrm{Fe}_{2} \mathrm{O}_{3}$ e queimadas em atmosfera de $\mathrm{CO} / \mathrm{CO}_{2}$. Quando o limite de solubilidade de $\mathrm{FeO}$ na fase liquida for alcançado, o $\mathrm{FeO}$ remanescente se manteve inalterado na sua fase (wustita). Porém, devido às fortes condições redutoras criadas no interior da cama-de-grafite durante a queima, todo o $\mathrm{FeO}$ presente nas amostras $\mathrm{P} 5 \mathrm{R}$ e P8R foi reduzido a $\alpha$-Fe, conforme mostrado na Equação F. Resultados semelhantes foram encontrados [18], onde foi observada a presença de $\alpha$-Fe em massas de louça sanitária queimadas em condições redutoras.

A Tabela III apresenta os resultados de absorção de água obtidos através da equação $\mathrm{A}$. As amostras sinterizadas em atmosfera de $\mathrm{O}_{2}$ apresentaram absorção de água próximo de zero. No entanto, para as formulações sinterizadas em atmosfera de $\mathrm{CO} / \mathrm{CO}_{2}$, somente a amostra P0R e P3R apresentaram absorção $<0,1 \%$. Para essa condição de queima, a crescente adição de $\mathrm{Fe}_{2} \mathrm{O}_{3}$ provocou um aumento na absorção de água. A micrografia da superfície polida das amostras contendo 0,3 e $8 \%$ peso $\mathrm{Fe}_{2} \mathrm{O}_{3}$ apresentado na Fig. 3 mostra um aumento na porosidade nas formulações contendo $\mathrm{Fe}_{2} \mathrm{O}_{3}$ e sinterizadas em atmosfera de $\mathrm{CO} / \mathrm{CO}_{2}$. Este aumento pode ser explicado pela presença de $\mathrm{CO}_{2}$ liberado durante a redução do $\mathrm{Fe}_{2} \mathrm{O}_{3}$, conforme descrito nas equações $\mathrm{D}$ a $\mathrm{F}$, o que explica o aumento de absorção de água para essas formulações.

A Fig. 4 apresenta as imagens obtidas por MEV das superfícies polidas e atacadas com $\mathrm{HF}$ das amostras contendo 0 e $3 \%$ peso de $\mathrm{Fe}_{2} \mathrm{O}_{3}$ sinterizadas em $\mathrm{O}_{2}$ e $\mathrm{CO} / \mathrm{CO}_{2}$. Todas as amostras apresentam grande quantidade da fase mulita secundaria, envolvidas em uma fase vítrea. Podemos observar que as amostras P0R e P3R (Figs. 4a e 4c, respectivamente) apresentam cristais de mulita mais alongados que as amostras $\mathrm{P} 0 \mathrm{O}$ e P3O (Figs. 4b e 4d, respectivamente). Esta diferença pode ser devido à baixa viscosidade da fase líquida provocada pela condição redutora, uma vez que, menores

Tabela III - Absorção de água para as formulações após sinterização em atmosfera oxidante e redutora.

[Table III - Water absorption for the formulations after the sintering in reducing and oxidizing atmosphere.]

\begin{tabular}{llcccc}
\hline Formulação $\left(\% p\right.$ de $\left.\mathrm{Fe}_{2} \mathrm{O}_{3}\right)$ & 0 & 3 & 5 & 8 \\
\hline Absorção de & Atmosfera $\mathrm{O}_{2}$ & 0,04 & 0,07 & 0,09 & 0,11 \\
\cline { 2 - 6 } \begin{tabular}{l} 
água (\%) \\
\cline { 2 - 6 }
\end{tabular} & Atmosfera $\mathrm{CO} / \mathrm{CO}_{2}$ & 0,01 & 0,10 & 0,36 & 0,93 \\
\hline
\end{tabular}



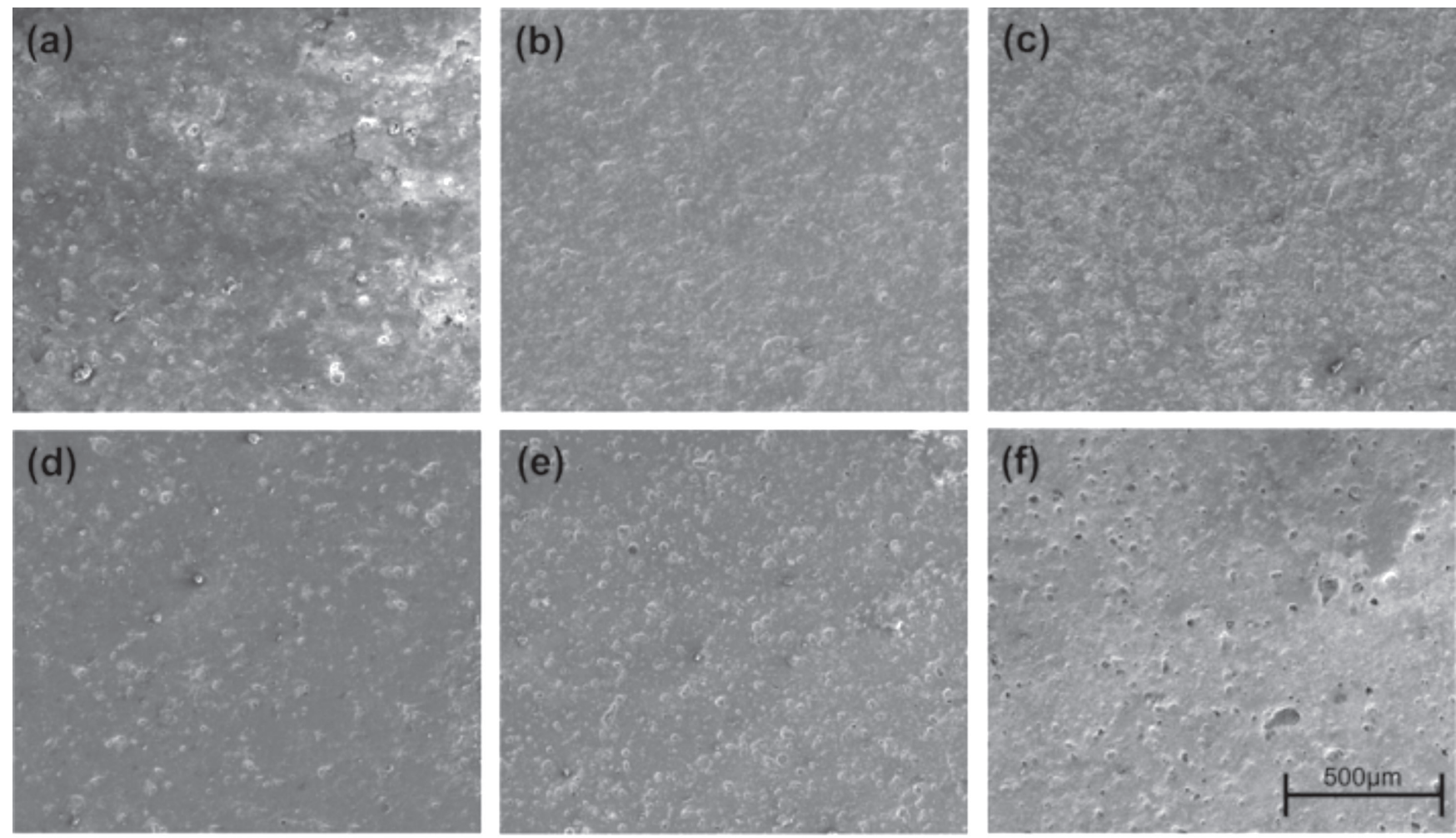

Figura 3: Micrografias obtidas em microscópio eletrônico de varredura da superfície polida das amostras: (a) P0O, (b) P3O, (c) P8O, (d) P0R, (e) P3R e (f) P8R.

[Figure 3: Scanning electron microscopy micrographs of the polished surface of samples (a) P0O, (b) P3O, (c) P8O, (d) P0R, (e) P3R and (f) P8R.]
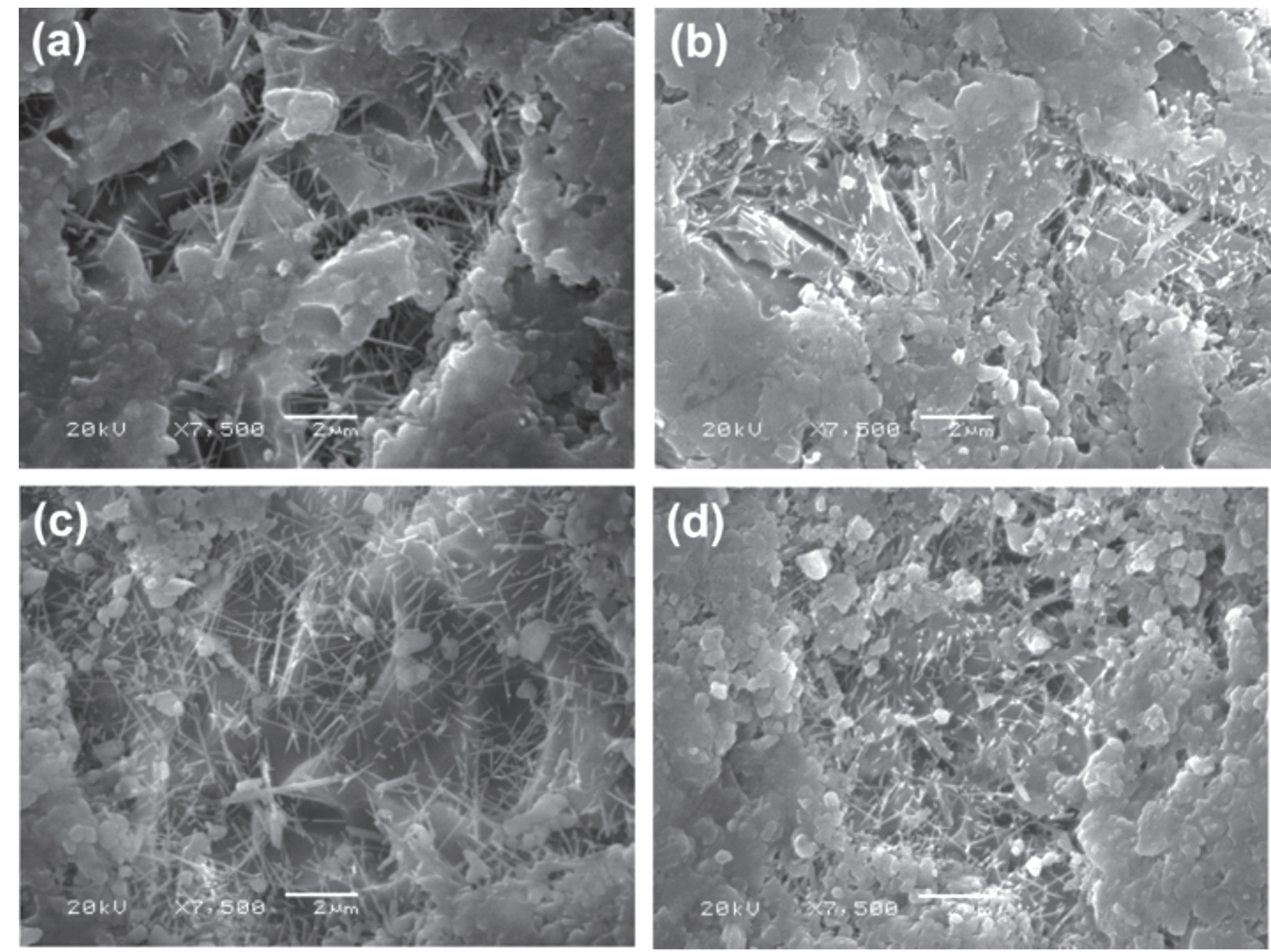

Figura 4: Micrografias obtidas em microscópio eletrônico de varredura da superfície polida e atacada com HF das amostras (a) P0R, (b) $\mathrm{P} 0 \mathrm{O}$, (c) P3R e (d) P3O.

[Figure 4: SEM micrographs of the polished and HF-etched for the samples (a) POR, (b) POO, (c) P3R and (d) P3O.] 
viscosidades aumentam a difusão das espécies envolvidas na cristalização da mulita e consequentemente, atenuam o crescimento do cristal. Além disso, para as duas condições (oxidante e redutora), a quantidade de cristais de mulita para as formulações contendo $3 \% \mathrm{p} \mathrm{de} \mathrm{Fe}_{2} \mathrm{O}_{3}$ é visualmente maior que as formulações contendo $0 \%$ peso de $\mathrm{Fe}_{2} \mathrm{O}_{3}$. Estudos em vidros de silicatos demonstram que, em condições oxidantes, $\mathrm{o} \mathrm{Fe}_{2} \mathrm{O}_{3}$ tende a dissolver-se na estrutura do vidro fornecendo íons $\mathrm{Fe}^{3+}$ [19]. Alguns íons de metais de transição, como o $\mathrm{Fe}^{3+}, \mathrm{Cr}^{3+}, \mathrm{Mn}^{3+}$, e $\mathrm{Ti}^{4+}$ podem favorecer a cristalização da mulita, substituindo o íon $\mathrm{Al}^{3+}$ em sua posição octaédrica na estrutura, chegando a formar uma solução sólida contendo até $13 \%$ p de $\mathrm{Fe}_{2} \mathrm{O}_{3}$ em elevadas temperaturas [20]. No entanto, os íons $\mathrm{Fe}^{2+}$ não apresentam essa mesma capacidade de mineralizador para a mulita devido a sua menor valência e maior raio iônico [21]. Desta forma, é de se esperar que grande parte da hematita tenha sido dissolvida na fase liquida antes de ser reduzida a $\mathrm{FeO}$. Assim, fornecendo grandes quantidades de $\mathrm{Fe}^{3+}$ que atuaram como mineralizadores para a mulita nas duas condições de queima.

A Fig. 5 apresenta os diagramas de impedância obtidos a $450{ }^{\circ} \mathrm{C}$. Essa temperatura foi escolhida por apresentar espectros bem definidos para todas as amostras. Inicialmente pode-se observar que todos os diagramas apresentam uma cauda em baixas frequências. Este fenômeno normalmente ocorre devido à polarização do eletrodo, efeito característico de condutores iônicos [22]. Os espectros para as amostras sintetizadas em atmosfera de $\mathrm{O}_{2}$ (Fig. 5a) apresentaram dois semicírculos pouco separados, enquanto apenas um único e achatado semicírculo é observado nas amostras sinterizadas em atmosfera $\mathrm{CO} / \mathrm{CO}_{2}$. Em trabalho sobre cerâmica à base de mulita-alumina foi observado apenas um semicírculo achatado, atribuído à continua fase vítrea do material [23] Utilizando espectroscopia de impedância para caracterizar a sinterização de cerâmica a base de argilas, foram encontrados resultados semelhantes, um único e achatado semicírculo, que também foi atribuído à fase vítrea [24]. Cada semicírculo em um diagrama de impedância é uma combinação de resistência-capacitância (RC) em paralelo [25] composition, ceramic texture, dopants and dopant or defect. Logo, fica evidente que a porcelana queimada em atmosfera de $\mathrm{O}_{2}$ possui duas regiões electroativas distintas que estão contribuindo separadamente para o comportamento global da amostra. Duas regiões também estão contribuindo para as amostras sinterizadas em atmosfera de $\mathrm{CO} / \mathrm{CO}_{2}$; no entanto, não estão separadas de forma clara devido à proximidade em suas frequências de relaxação.

Uma das principais vantagens da técnica de espectroscopia de impedância é que podemos atribuir os resultados dos espectros a regiões na amostra (grãos, contornos de grão, interfaces), podendo assim verificar a contribuição dessas regiões no comportamento global do material [26]. Uma maneira utilizada para identificar essas regiões é por meio
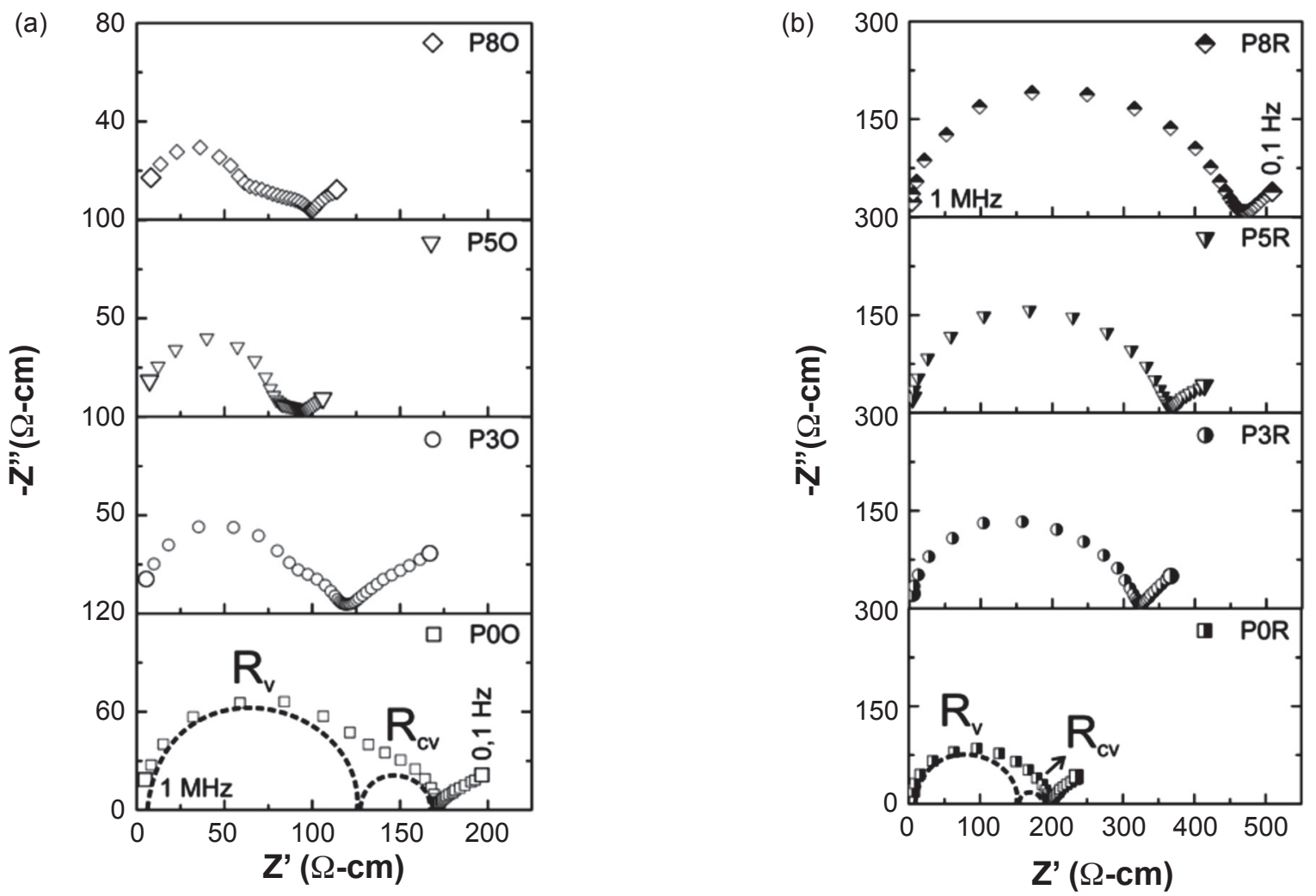

Figura 5: Diagramas de impedância obtidos a $450{ }^{\circ} \mathrm{C}$ para todas as formulações sinterizadas em atmosfera of (a) $\mathrm{O}_{2}$ e (b) $\mathrm{CO} / \mathrm{CO}_{2}$. [Figure 5: Impedance diagrams at $450{ }^{\circ} \mathrm{C}$ for all the formulations sintered at atmosphere of (a) $\mathrm{O}_{2}$ and (b) $\mathrm{CO} / \mathrm{CO}_{2}$.] 

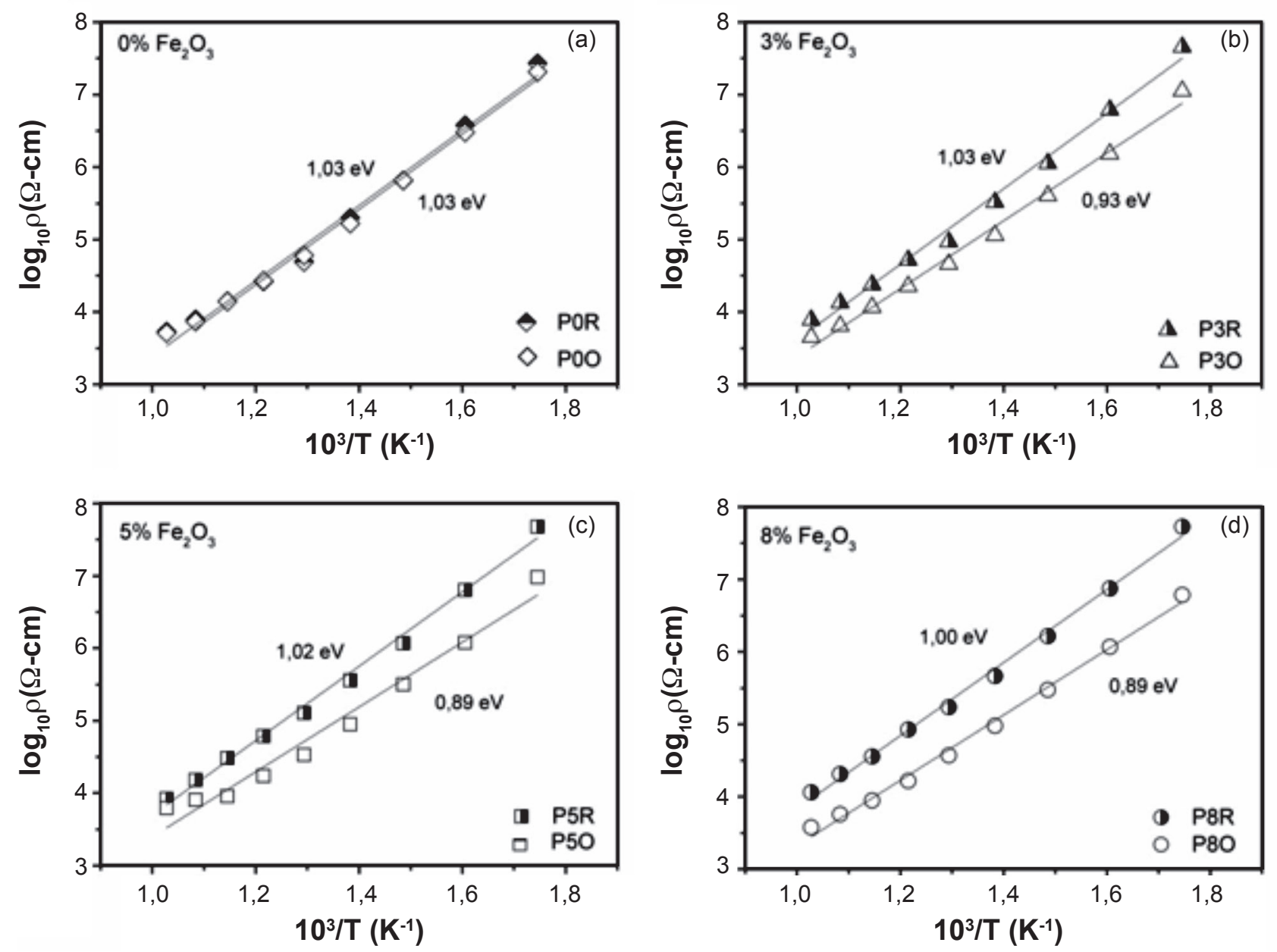

Figura 6: Gráficos de Arrhenius da resistividade das amostras contendo diferentes concentrações de $\mathrm{Fe}_{2} \mathrm{O}_{3}$ e sinterizadas em $\mathrm{O}_{2}$ e $\mathrm{CO} / \mathrm{CO}_{2}$. (a) $0 \%$ peso de $\mathrm{Fe}_{2} \mathrm{O}_{3}$, (b) $3 \%$ peso de $\mathrm{Fe}_{2} \mathrm{O}_{3}$, (c) $5 \%$ peso de $\mathrm{Fe}_{2} \mathrm{O}_{3}$ e (d) $8 \%$ peso de $\mathrm{Fe}_{2} \mathrm{O}_{3}$.

[Figure 6: Arrhenius plots of the resistivity of samples containing different $\mathrm{Fe}_{2} \mathrm{O}_{3}$ concentrations and sintered under $\mathrm{O}_{2}$ and $\mathrm{CO} / \mathrm{CO}$. (a) $\mathrm{O}$ $w t . \% \mathrm{Fe}_{2} \mathrm{O}_{3}$, (b) $3 w t . \% \mathrm{Fe}_{2} \mathrm{O}_{3}$, (c) $5 w t . \% \mathrm{Fe}_{2} \mathrm{O}_{3}$ and (d) $\left.8 w t . \% \mathrm{Fe}_{2} \mathrm{O}_{3}\right]$.

da capacitância (C) associada aos semicírculos. Baixos valores de capacitância estão associados a fases com grandes frações volumétricas ("bulk" do material), enquanto que elevados valores estão normalmente associados a menores regiões (interfaces e contornos de grão) [25]. As capacitâncias associadas a diferentes semicírculos encontrados nos diagramas de impedância a $450{ }^{\circ} \mathrm{C}$ foram determinadas por meio da relação $\mathrm{C}=1 / 2 \pi f \mathrm{R}$, na qual $f$ é a frequência de relaxação no apex do semicírculo e $\mathrm{R}$ a resistência. Os resultados indicam que os semicírculos de alta frequência para todas as amostras possuem baixa capacitância $\left(\sim 10^{-12} \mathrm{~F}\right)$, enquanto que os semicírculos em baixa frequência apresentam relativa elevada capacitância $\left(\sim 10^{-9}\right.$ F). Considerando a classificação proposta [25] composition, ceramic texture, dopants and dopant (or defect e os valores de capacitância aqui calculados, o semicírculo em alta frequência foi atribuído à fase vítrea $\left(\mathrm{R}_{\mathrm{v}}\right)$, já que ela é a fase predominante em uma porcelana, enquanto que o semicírculo em baixa frequência foi atribuído às regiões de interface entre fase vítrea e fases cristalinas $\left(\mathrm{R}_{\mathrm{vC}}\right)$. Uma vez definido semicírculo do diagrama com uma região da amostra, é possível observar que para todas as formulações, a resistividade da fase vítrea domina a resistividade total da amostra. Além disso, a adição de $\mathrm{Fe}_{2} \mathrm{O}_{3}$ na formulação STD resultou em um efeito oposto na resistividade elétrica das porcelanas sinterizadas em atmosfera de $\mathrm{O}_{2}$ e $\mathrm{CO} / \mathrm{CO}_{2}$. Para a condição oxidante (Fig. 5a), a adição de $\mathrm{Fe}_{2} \mathrm{O}_{3}$ provocou uma diminuição em $\mathrm{R}_{\mathrm{v}} \mathrm{e} \mathrm{R}_{\mathrm{vC}}$. No caso da atmosfera redutora (Fig. 5b), o $\mathrm{Fe}_{2} \mathrm{O}_{3}$ adicionado à formulação resultou em um aumento em ambos os valores de $\mathrm{R}_{\mathrm{v}} \mathrm{e} \mathrm{R}_{\mathrm{VC}}$. A diminuição da resistividade total para as amostras contendo $\mathrm{Fe}_{2} \mathrm{O}_{3}$ e sinterizadas em atmosfera de $\mathrm{O}_{2}$ pode estar relacionada à presença da fase altamente condutora hematita não solubilizada. A presença da fase hematita mostra que há uma elevada concentração de íons de ferro dispersos na fase vítrea, o que pode provocar o surgimento de pares $\mathrm{Fe}^{2+}-\mathrm{Fe}^{3+}$ e possibilitando que a condução por small polaron hopping entre esses íons aconteça. Além disso, recentes investigações em vidros contendo ferro demonstraram que as regiões de interface criadas durante o processo de devitrificação a partir de uma fase liquida rica em íons de ferro foram cruciais para o aumento da condutividade [27]. Foi mostrado que 
nas regiões interfaciais a concentração de pares $\mathrm{Fe}^{2+}$ e $\mathrm{Fe}^{3+}$ é superior à do interior da matriz vítrea, formando assim um caminho para a condução de elétrons nessa região. Isto pode explicar a diminuição de $\mathrm{R}_{\mathrm{vC}}$ com a adição de $\mathrm{Fe}_{2} \mathrm{O}_{3}$ nas amostras sinterizadas em $\mathrm{O}_{2}$, uma vez que grande parte da mulita foi cristalizada a partir de uma fase liquida rica com íons de ferro.

Conforme discutido anteriormente nos resultados de difração de raios $X$ apresentados na Fig. 2b, o limite de solubilidade para a amostra contendo inicialmente concentrações acima de $3 \%$ peso de $\mathrm{Fe}_{2} \mathrm{O}_{3}$ e sinterizadas em atmosfera de $\mathrm{CO} / \mathrm{CO}_{2}$ foi alcançado, fazendo surgir a fase $\alpha$-Fe. Embora essa fase possua uma elevada condutividade, seu efeito não foi pronunciado na diminuição da resistividade da amostra P5R e P8R. Uma possível explicação para esse comportamento pode ser baseada nos resultados reportados [28]. Nesse trabalho sobre a presença de partículas de ferro em vidros, é afirmado que a condução elétrica ocorrerá ao longo de um caminho no qual a energia de ativação é mínima. No entanto, a fase condutora deve apresentar um caminho razoável para que a condução ocorra. Logo, a elevada presença de fase vítrea nas amostras P5R e P8R pode ter envolvido completamente as partículas de ferro, não permitindo que a condução entre ambas ocorresse. A diminuição nos picos da fase quartzo (Fig. 2b) reforça a ideia da formação de elevadas concentrações de fase liquida durante a queima para as amostras P3R, P5R e P8R. Além disso, as imagens (Fig. 3) mostram um aumento pronunciado na porosidade para a amostra contendo inicialmente em sua formulação $\mathrm{Fe}_{2} \mathrm{O}_{3}$ e sinterizada em atmosfera de $\mathrm{CO} / \mathrm{CO}_{2}$. Foi considerado que em um material totalmente denso as linhas de corrente são preferencialmente paralelas ao campo elétrico aplicado [29]. No entanto a presença de poros faz com que a corrente tenha que contorná-los, requerendo maior caminho para atravessar o material, fazendo dessa forma a sua resistência aumentar. Dessa forma, a elevada presença de poros pode ter contribuído para o aumento de $\rho$ nas amostras sinterizadas em atmosfera de $\mathrm{CO} / \mathrm{CO}_{2}$.

A dependência da $\rho$ com a temperatura para todas as amostras é representada em um gráfico tipo Arrhenius na Fig. 6. Devido às amostras terem apresentado em baixa temperatura resistência elétrica superior ao limite do equipamento (20 $\mathrm{M} \Omega$ para este caso), somente medidas em temperaturas acima de $300{ }^{\circ} \mathrm{C}$ foram coletadas. Para essa faixa de estudo $\left(350-750{ }^{\circ} \mathrm{C}\right)$ fica evidente nos gráficos da Fig. 6 uma redução em $\rho$ com o aumento da temperatura para todas as amostras, chegando a cair até 4 ordens de grandeza em $400{ }^{\circ} \mathrm{C}$. Esta diminuição mostra que o mecanismo de condução para as porcelanas aqui sob estudo é um processo termicamente ativado, estando em acordo com outros estudos de porcelanas elétricas $[9,30]$. De acordo com a equação $\sigma=n_{e} e \mu$ a condutividade elétrica é expressa em função da concentração de portadores de carga $\left(n_{e}\right)$, carga desses portadores $(e)$ e a sua mobilidade $(\mu)$. Os valores de $e$ não dependem da temperatura, enquanto $n_{e} \mathrm{e} \mu$ são altamente dependentes. Com o aumento da temperatura haverá um maior número de portadores de carga e um aumento em sua mobilidade, o que leva a um aumento da condutividade ou, em outras palavras, uma diminuição da resistividade como o apresentado pelas porcelanas aqui investigadas. Não obstante, deve-se considerar que cada fase na porcelana terá sua influência específica sobre a resistividade global. A resistividade da mulita a temperatura ambiente é de aproximadamente $10^{13} \Omega . \mathrm{cm}$, no entanto sua resistividade cai notavelmente com o aumento da temperatura, comportandose como um semicondutor em altas temperaturas [31]. A fase vítrea também possui a mesma dependência com a temperatura. Originada a partir da fusão de feldspatos, a fase vítrea possui grande concentração de cátions $\left(\mathrm{Na}^{+} \mathrm{e} \mathrm{K} \mathrm{K}^{+}\right.$), que com o aumento da temperatura, promovem um aumento na condutividade elétrica [32].

Nos gráficos de Arrhenius (Fig. 6) também é possível observar que para todas as formulações contendo $\mathrm{Fe}_{2} \mathrm{O}_{3}$, as amostras sinterizadas em atmosfera de $\mathrm{CO} / \mathrm{CO}_{2}$ apresentaram maiores valores de $\rho$. Conforme descrito anteriormente, a elevada quantidade de fase vítrea e a presença de poros para as amostras sinterizadas em $\mathrm{CO} / \mathrm{CO}_{2}$ e a presença da fase hematita nas amostras sinterizadas em $\mathrm{O}_{2}$ podem ser considerados como os principais fatores responsáveis por este comportamento. A atmosfera de queima praticamente não teve influência apenas no caso da formulação não contendo $\mathrm{Fe}_{2} \mathrm{O}_{3}$. Os valores da energia de ativação para as formulações sinterizadas nas duas atmosferas são apresentadas junto aos gráficos na Fig. 6. Esses valores foram calculados a partir da inclinação das curvas por meio da equação $\mathrm{C}$. Como mostrado, a $\mathrm{E}_{\mathrm{a}}$ exibiu uma tendência em reduzir seu valor com o aumento da adição de $\mathrm{Fe}_{2} \mathrm{O}_{3}$ para as amostras sinterizadas em condição oxidante, enquanto que para as condições redutoras, a $E_{a}$ se manteve praticamente inalterada. Os valores de $\mathrm{E}_{\mathrm{a}}$ próximos a $1,0 \mathrm{eV}$ estão em concordância com valores reportados de 1,08 a $1,33 \mathrm{eV}$ [9]. As amostras contendo elevada concentração de $\mathrm{Fe}_{2} \mathrm{O}_{3}$ e sinterizadas em $\mathrm{O}_{2}$ apresentaram menor valor de $\mathrm{E}_{\mathrm{a}}$, porém esses resultados ainda estão próximos aos de 0,79 a $0,87 \mathrm{eV}$ [30]. Em ambas referências é afirmado que embora as porcelanas sejam consideradas isolantes na temperatura ambiente, em elevadas temperaturas se comportam como semicondutores. Este fato está de acordo com os resultados encontrados neste trabalho, no qual, a $400{ }^{\circ} \mathrm{C}$ as amostras exibiram em média uma resistividade de $10^{4,5}$ a $10^{5,5} \Omega . \mathrm{cm}$, valores similares aos encontrados para alguns materiais semicondutores [33].

\section{CONCLUSÕES}

Porcelanas aluminosas contendo diferentes concentrações de $\mathrm{Fe}_{2} \mathrm{O}_{3}$ foram sinterizadas em atmosferas oxidante e redutora para avaliação de seu comportamento elétrico. A adição de $\mathrm{Fe}_{2} \mathrm{O}_{3}$ resultou em um aumento na concentração de mulita, devido ao efeito do $\mathrm{Fe}^{3+}$ como mineralizador. Além disso, a sinterização em atmosfera de $\mathrm{CO} / \mathrm{CO}_{2}$ provocou o crescimento dos cristais de mulita. Os diagramas de impedância mostraram que duas regiões contribuem com maior intensidade para as propriedades 
elétricas das porcelanas aluminosas: a fase vítrea e as interfaces entre a fase vítrea e fases cristalinas. Para a condição oxidante, a resistividade elétrica diminui com a adição de $\mathrm{Fe}_{2} \mathrm{O}_{3}$ devido à presença de hematita. De modo contrário, em atmosfera redutora a adição de $\mathrm{Fe}_{2} \mathrm{O}_{3}$ resultou em um aumento na resistividade elétrica das amostras. Esse aumento foi atribuído à elevada quantidade de fase vítrea e porosidade. Portanto, atmosferas redutoras durante a queima podem favorecer o uso de matérias-primas com elevadas concentrações de $\mathrm{Fe}_{2} \mathrm{O}_{3}$, desde que esta concentração não exceda o limite de solubilidade deste óxido na fase liquida durante a sinterização.

\section{AGRADECIMENTOS}

Os autores gostariam de agradecer a FAPESP e a CAPES pelo auxilio para a execução da pesquisa.

\section{REFERÊNCIAS}

[1 R. C. Buchanan, "Ceramic Materials for Electronics Processing, Properties, and Applications", $2^{\text {nd }}$ ed., New York, EUA (1986) .

[2] W. M. Cam, U. Senapati, J. Am. Ceram. Soc. 81 (1998) 3.

[3] J. M. Amigó, F. J. Serrano, M. A. Kojdecki, J. Bastida, V. Esteve, M. M. Reventós, F. Marti, J. Eur. Ceram. Soc. 25 (2005) 1479.

[4] Y. Iqbal, W. E. Lee, J. Am. Ceram. Soc. 90 (1999) 3584.

[5] R. E. Hummel, "Electronic properties of materials", New York, EUA (1895).

[6] J. Liebermann, Am. Ceram. Soc. Bull. 83 (2003) 39.

[7] N. Montoya, F. J . Serrano, M. M. Reventós, J. M. Amigo, J. Alarcón, J. Eur. Ceram. Soc. 30 (2010) 839.

[8] S. P. Chaudhuri, P. Sarkar, Ceram. Int. 26 (2000) 865.

[9] S. P. Chaudhuri, P. Sarkar, A. K. Chakraborty, Ceram. Int. 25 (1999) 91.

[10] Z. D. Taszic, J. Mater. Sci. 28 (1993) 5693.

[11] R. H. Piva, P. Vilarinho, M. R. Morelli, M. A. Fiori, O. R. K. Montedo, Ceram. Int. 39 (2013) 7323.

[12] E. Kamseu, C. Leonelli, D. N. Boccaccini, P. Veronesi, P. Miselli, G. Pellacani, U. C. Melo, Ceram. Int. 33 (2007) 851.
[13] A. L. Chinelatto, D. P. F. de Souza, Cerâmica 50 (2004) 62.

[14] R. Chand, E. Obuchi, K. Katoh, H. N. Luitel, K. Nakano, Catal. Comm. 13 (2011) 49

[15] P. W. Olupot, S. Jonsson, J. K. Byaruhanga, Ceram. Int. 36 (2010) 1455.

[16] J. Liebermann, Interceram. 57 (2008) 247.

[17] H. D. H. Du, H. X. H. Xiao, Second Int. Conf. Prop. Appl. Diel. Mater., Beijing, China 1 (1988) 121.

[18] G. P. Souza, P. F. Messer, W. E. Lee, J. Am. Ceram. Soc. 89 (2006) 1993.

[19] E. S. Dunaeva, I.A. Uspenskaya, K. V. Pokholok, V. V. Minin, N. N. Efimov, E. A. Ugolkova, E. Brunet, J. NonCryst.e Solids 358 (2012) 3089.

[20] S. Ilić, S. Zec, M. Miljković, D. Poleti, M. Pošarac -Marković, D. Janaćković, Dj. Janaćković, J. Alloy. Compd. 612 (2014) 259.

[21] S. P. Chaudhuri, S. K. Patra, A. K. Chakraborty, J. Eur. Ceram. Soc. 19 (1999) 2941.

[22] J. E. Garbarczyk, M. Wasiucionek, P. MacHowski, W. Jakubowski, Solid State Ionics 119 (1999) 9.

[23] M. J. Ribeiro, J. C. C. Abrantes, J. M. Ferreira, J. A. Labrincha, J. Eur. Ceram. Soc. 24 (2004) 3841.

[24] X. Wang, P. Xiao, J. Eur. Ceram. Soc. 22 (2002) 471.

[25] J. T. S. Irvine, D.C. Sinclair, A. R. West, Adv. Mater. 2 (1990) 132.

[26] D. C. Sinclair, Bol. Soc. Esp. Ceram. y Vidrio 65 (1995) 55.

[27] J. E. Garbarczyk, M. Wasiucionek, P. Jozwiak, J. L. Nowinski, C. M. Julien, Solid State Ionics. 180 (2009) 531.

[28] S. Roy, D. Chakravorty, J. Mater. Res. 9 (1994) 2314.

[29] P. J. Panteix, I. Julien, P. Abélard, D. Ceram. Int. 34 (2008) 1579.

[30] A. Ş. Demirkiran, R. Artir, E. Avci, Ceram. Int. 36 (2010) 917

[31] M. Malki, C. M. Hoo, M. L. Mecartney, H. Schneider, J. Am. Ceram. Soc. 97 (2014) 1923.

[32] M. L. Braunger, C. A. Escanhoela, I. Fier, L. Walmsley, E. C. Ziemath, J Non-Crystalline Solids. 358 (2012) 2855.

[33] W. D. Callister, Ciência e Engenharia dos Materiais: uma Introdução, $5^{a}$ ed., John Wiley \& Sons, New York, EUA (2000) p. 417.

(Rec. 10/03/2015, Ac. 08/05/2015) 